mutant isoform. This interpretation is supported by the observation that, in both worms and mammalian cells, the effects of dominant forms of RME-1 were compensated by expression of additional copies of the wild-type protein ${ }^{7,8}$.

Interestingly, although there is a recycling endosome compartment ${ }^{16,17}$ in budding yeast, its genome lacks a member of the RME-1 gene family. Thus, RME-1 may perform a specialized function that occurs only in higher eukaryotes. Alternatively, proteins with functional homology or unrelated factors may exist that regulate membrane recycling in yeast and other organisms.

Important questions remain, such as the identification of RME-binding molecules, both in the cytoplasm and on the endosome membrane. As EH domains are well-characterized protein-binding modules, most of which recognize Asn-Pro-Phe motifs, it will be of great interest to learn whether RME-1 association with the endosome is mediated by its EH domain. For instance, the RME-1 'receptor' might be an Asn-Pro-Phe motifcontaining membrane protein. Alternatively, the EH domain ligand may be a protein that bridges interactions between RME- 1 and an endosome-membrane protein. It will also be of great interest to know how the dominant negative $\mathrm{EH}$ domain mutant protein affects endosomal function. Is it altering the function of other $\mathrm{EH}$ domain-containing proteins or altering interactions of other domains of RME-1? Finally, it will be interesting to find out the mechanism that requires RME-1 for membrane transport through the ERC. Whereas exit from the ERC is thought to be a default pathway, it nonetheless requires a cellular machinery to effect the movements of proteins into vesicles or tubules and to direct them to the plasma membrane. Further studies of how RME-1 acts to control exit from the ERC will provide important information about a process that is central to ensuring adequate nutrient uptake through receptor recycling.

Beverly Wendland is in the Department of Biology,
Johns Hopkins University, 3400 N Charles street, Baltimore, Maryland 21218, USA.

e-mail: bwendland@jhu.edu

1. Mukherjee, S., Ghosh, R. N. \& Maxfield, F. R. Endocytosis Physiol. Rev. 77, 759-803 (1997).

2. Yamashiro, D. J., Tycko, B., Fluss, S. R. \& Maxfield, F. R. Cell 37, 789-800 (1984).

3. Zuk, P. A. \& Elferink, L. A. J. Biol. Chem. 274, 22303-12 (1999).

4. Ullrich, O., Reinsch, S., Urbe, S., Zerial, M. \& Parton, R. G. J. Cell Biol. 135, 913-24 (1996).

5. Prekeris, R., Klumperman, J., Chen, Y. A. \& Scheller, R. H. J. Cell Biol. 143, 957-71 (1998).

6. Daro, E., van der Sluijs, P., Galli, T. \& Mellman, I. Proc. Natl Acad. Sci. USA 93, 9559-64 (1996).

7. Lin, S. X., Grant, B., Hirsh, D. \& Maxfield, F. R. Nature Cell Biol. 3, 567-572 (2001).

8. Grant, B. et al. Nature Cell Biol. 3, 573-579 (2001).

9. Saraste, M., Sibbald, P. R. \& Wittinghofer, A. Trends Biochem. Sci. 15, 430-4 (1990).

10. Santolini, E., Salcini, A. E., Kay, B. K., Yamabhai, M. \& Di Fiore, P. P. Exp. Cell Res. 253, 186-209 (1999).

11. Pohl, U. et al. Genomics 63, 255-62 (2000).

12. Haider, N. B. et al. Gene 240, 227-32 (1999).

13. Torrisi, M. R. et al. Mol. Biol. Cell 10, 417-34 (1999).

14. Grant, B. \& Hirsh, D. Mol. Biol. Cell 10, 4311-26 (1999).

15. Fire, A. et al. Nature 391, 806-11 (1998).

16. Chen, L. \& Davis, N. G. J. Cell Biol. 151, 731-8 (2000).

17. Wiederkehr, A., Avaro, S., Prescianotto-Baschong, C., Haguenauer-Tsapis, R. \& Riezman, H. J. Cell Biol. 149, 397-410 2000).

\title{
Passage through the nuclear pore
}

Transport into and out of the nucleus is fundamental for many aspects of cellular function. Cargo molecules are transported through nuclear pore complexes (NPCs). Small molecules can pass by diffusion, but there is a permeability barrier for larger molecules, with a relative molecular mass of $>20-40 \mathrm{~K}$, which permits transport of only selective cargo with the help of specific nuclear transport receptors. However, the nature of this barrier and the precise molecular mechanism for selective transport are largely unknown. In a recent paper (EMBO J. 20, 1320-1330, 2001), Ribbeck and Görlich provide an intriguing model, the 'selective-phase hypothesis' which integrates both aspect of translocation.

In a system optimised to avoid other rate-limiting factors, the authors measured the translocation capacity of NPCs using various model substrates, and observed maximal translocation rates on the order of $10^{3}$ translocations per second, which is much higher than previously thought. For a homodimer of the nuclear transport receptor NTF2, for example, this rate is relatively close to the free diffusion rate through a hypothetical NPC lacking a permeability barrier. In contrast, translocation of green fluorescent protein (GFP), which has a similar molecular size, is about 100 -fold slower.

How can this difference be explained? It is known that translocation facilitated by nuclear transport receptors requires that the receptors interact with phenylalanine (Phe)-rich repeats found in high numbers on many NPC components. The concentration of these repeats in the nuclear pore is estimated to be as high as $50 \mathrm{mM}$. Ribbeck and Görlich propose that these repeats (light blue circles) form weak hydrophobic interactions with each other and constitute the molecular equivalent of the so-called central plug, a low electron density structure that fills electron micrographs of nuclear pores. Thus a sieve-like structure is formed that allows the diffusion of small molecules but prevents the passage of larger ones. In this hypothesis, nuclear
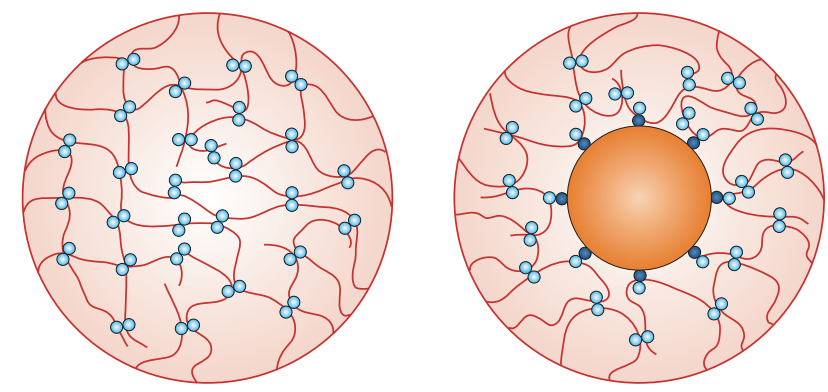

THE EMBO JOURNAL

transport receptors (orange circle), through their ability to interact with these repeats (dark blue circles), partition into and pass through this meshwork. As the hydrophobic interactions between Phe-repeats are relatively weak, nuclear transport receptors would only require micromolar affinities to disrupt Pherepeat interactions and thereby pass through the central plug. Such low affinity interactions and the corresponding high offrates are consistent with the speed of nuclear transport.

Intriguingly, this model mirrors the concept of lipid bilayers, which also exhibit a permeability barrier and allow only the passage of molecules that can partition into the hydrophobic core of the bilayer. Although further work is required to provide experimental support for the 'selective-phase hypothesis', it is an exciting step forward in providing a model for both the molecular nature of the permeability barrier and for the selective ability of nuclear transport receptors to pass through NPCs.

BARBARA MARTE 